OPEN ACCESS

Edited by:

Huan-Hsuan Hsu,

Tufts University, United States

Reviewed by:

Chunsheng Wu,

Xi'an Jiaotong University, China

Jin Wu,

Sun Yat-sen University, China

${ }^{*}$ Correspondence:

Xing Chen

cnxingchen@zju.edu.cn

Di Wang

diwang@zhejianglab.com

Specialty section:

This article was submitted to Biosensors and Biomolecular

Electronics,

a section of the journal

Frontiers in Bioengineering and

Biotechnology

Received: 25 January 2022 Accepted: 14 February 2022

Published: 08 March 2022

Citation:

Dong H, Qian L, Cui Y, Zheng X, Cheng C, Cao Q, Xu F, Wang J, ChenX and Wang $D$ (2022) Online Accurate Detection of Breath Acetone Using

Metal Oxide Semiconductor Gas Sensor and Diffusive Gas Separation. Front. Bioeng. Biotechnol. 10:861950.

doi: 10.3389/fbioe.2022.861950

\section{Online Accurate Detection of Breath Acetone Using Metal Oxide Semiconductor Gas Sensor and Diffusive Gas Separation}

\author{
Hao Dong ${ }^{1,2}$, Libin Qian ${ }^{1}$, Yaoxuan Cui ${ }^{1}$, Xubin Zheng ${ }^{1}$, Chen Cheng ${ }^{1}$, Qingpeng Cao ${ }^{1}$, \\ Feng $\mathrm{Xu}^{1}{ }^{1}$, Jin Wang ${ }^{1}$, Xing Chen ${ }^{2 *}$ and Di Wang ${ }^{1 *}$ \\ ${ }^{1}$ Intelligent Perception Research Institute, Zhejiang Lab, Hangzhou, China, ${ }^{2}$ Key Laboratory for Biomedical Engineering of \\ Education Ministry of China, Zhejiang University, Hangzhou, China
}

Breath acetone (BrAce) level is an indicator of lipid oxidation rate, which is crucial for evaluating the status of ketoacidosis, ketogenic diet, and fat burning during exercise. Despite its usefulness, detecting BrAce accurately is challenging because exhaled breath contains an enormous variety of compounds. Although many sensors and devices have been developed for BrAce measurement, most of them were tested with only synthetic or spiked breath samples, and few can detect low concentration BrAce in an online manner, which is critical for extending application areas and the wide acceptance of the technology. Here, we show that online detection of BrAce can be achieved using a metal oxide semiconductor acetone sensor. The high accuracy measurement of low concentration BrAce was enabled by separating major interference gases utilizing their large diffusion coefficients, and the accuracy is further improved by the correction of humidity effect. We anticipate that the approach can push BrAce measurement closer to being useful for various applications.

Keywords: acetone, MOS sensor, breath analysis, diffusive gas separation, ketogenic diet

\section{INTRODUCTION}

Human excretes more than 200 volatile organic compounds (VOCs) through respiration, which contains many physiological and biochemical information (Pauling et al., 1971; Phillips et al., 1999). Due to the noninvasive nature, breath analysis has been considered to be a promising method for disease diagnosis and health management (Chen et al., 2017; Dong et al., 2017; Dong et al., 2020; Chen et al., 2021). An increasing number of breath-based tests have already been extensively employed in clinical, e.g., Helicobacter pylori test (Stefano et al., 2018), etc.

Among all these VOCs, Breath acetone (BrAce), as a by-product of the fat metabolism process, has been measured to monitor ketosis in healthy and diabetic subjects (Wang and Wang, 2013; Alizadeh et al., 2019). It has been shown that BrAce level can go up to $100 \mathrm{ppm}$ for individuals with ketogenic diet and fasting conditions and increase up to $1250 \mathrm{ppm}$ for poorly controlled diabetes with ketoacidosis. In contrast to these high BrAce conditions, BrAce of normal healthy individuals usually ranges from only 0.5-2.0 ppm (Anderson, 2015). The BrAce fluctuation within this range has been shown to provide insight into an individual's lifestyle, level of hunger, and efficiency of work out (Musa-Veloso et al., 2002; King et al., 2009; Španěl et al., 2011). Thus, accurate detection of low concentration BrAce will enable the management of daily diet and exercise, alerting lifestyle and preventing the onset of diseases. 

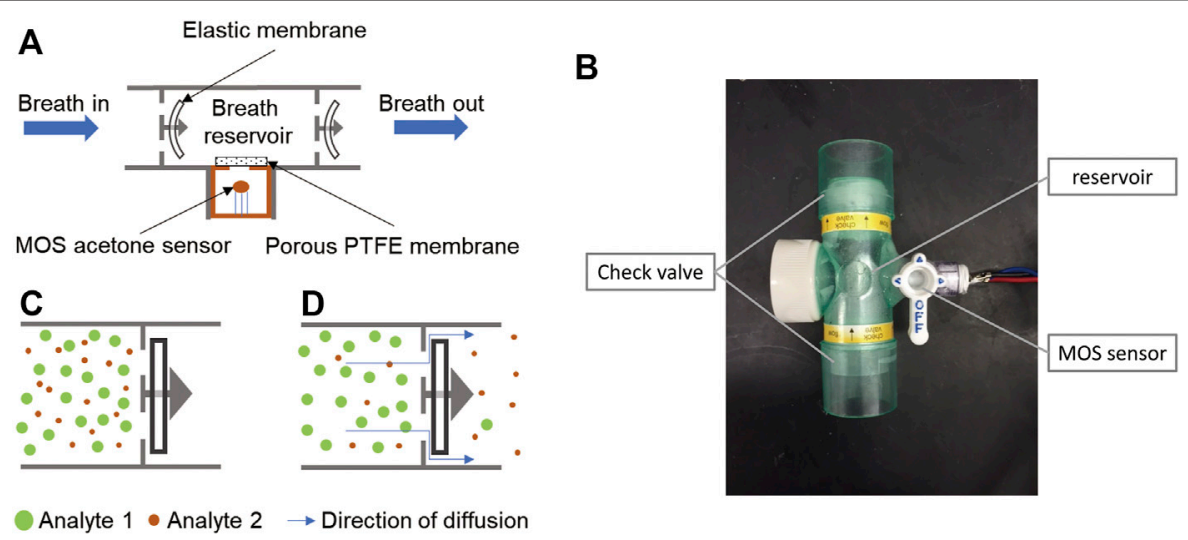

FIGURE 1 | Principle and structure of the diffusive gas separation device for BrAce measurement. (A) During the sampling process, human subjects directly breathe into the chamber. The elastic membranes are bent by the breath pressure, allowing the exhalation to pass through. (B) When sampling stops, the two elastic membranes restore to their original flat shape and the breath sample is trapped. (C) The molecules of exhalation can escape from the reservoir via small gaps between the elastic membrane and its holder, while the escape speed of different molecules varied with their diffusion coefficients. (D) The image of the diffusive gas separation device.

The traditional methods for trace BrAce detection employ chromatography and mass spectrometry-based standard equipment (Schwarz et al., 2009; Španěl et al., 2011). However, considering cost, size, and complexity, these methods are not practical for people to monitor their fat metabolisms frequently at different places. Chemical sensors are attractive alternatives due to their small size, low cost, and easy operation (Wu et al., 2021; Liang et al., 2022). Different types of chemical sensors have been developed for BrAce detection. Colorimetric sensors have good sensitivity and selectivity (Wang et al., 2020). However, they are typically irreversible and for one-time use only, which increases the cost of frequent use. Lots of efforts have been devoted to the development of metal oxide semiconductor (MOS) acetone sensors (Shin et al., 2013; Koo et al., 2017; Cho et al., 2020; Chen et al., 2022). MOS sensors are small, sensitive, and reusable, but the selectivity issue compromises their performance in the test of complex breath samples. Although lots of works on MOS acetone sensors have been published, very few demonstrated detection of real breath samples with good accuracy, especially for online direct breath test without using a sampling bag, which is critical for the practicality and widespread adoption of the technology.

In this work, we show that online accurate detection of BrAce can be achieved using a MOS sensor via diffusive separation of gaseous compounds. A breath reservoir with two check valves is used for both comfort breath sampling and gas separation. Although the MOS sensor is sensitive to many compounds in the breath, interference gases like hydrogen escape from the breath reservoir faster than acetone because of larger diffusion coefficients. We optimized the diffusion time, corrected humidity interference, calibrated the device using real breath samples against a standard instrument, and demonstrated its performance under realistic application scenarios.

\section{MATERIALS AND METHODS}

\section{Materials and Apparatus}

MOS acetone sensors based on platinum doped tungsten oxide (SB-33) were purchased from FIS, Inc. Non-rebreathing T-pieces were purchased from Vacumetrics, Inc. Teflon membranes (VE8) were purchased from Membrane Solutions, Inc. The humidity sensor (HIH-5030-001) was purchased from Honeywell, Inc.

\section{Device Configuration}

The assembly diagram of the diffusive gas separation device is shown in Figure 1A, a non-rebreathing T-piece with two check valves is used for breath sampling and gas separation. The MOS sensor is placed in the reservoir for acetone sensing. Direct airflow on the sensor surface will change the conductivity of the sensor by altering the heated layer temperature. Therefore, we placed a porous Teflon membrane between the reservoir and the sensor. The membrane allows diffusion of the gases, while reducing the influence of the air speed. In addition, a humidity sensor is integrated into the reservoir for humidity monitoring and correction.

\section{BrAce Measurements}

When the commercial MOS sensor (SB-33) is exposed to acetone, acetone reacts with oxygen ions adsorbed on the surface of the platinum doped tungsten oxide, resulting in electron transfer and the impedance change of the sensor.

The MOS sensor resistance was detected using a STM32 MCU based module integrated with an AD7792 A/D converter. We monitored the sensor baseline $\left(\mathrm{R}_{\mathrm{air}}\right)$ for $20 \mathrm{~s}$ before tests, and the response of the MOS sensor was defined as the ratio of resistance $\left(R_{\mathrm{air}} / R\right)$.

In addition, the concentrations of BrAce were validated using a selected ion flow tube-mass spectrometer (SIFT-MS) (Instrument Science, Profile Series, Crewe, United Kingdom) 

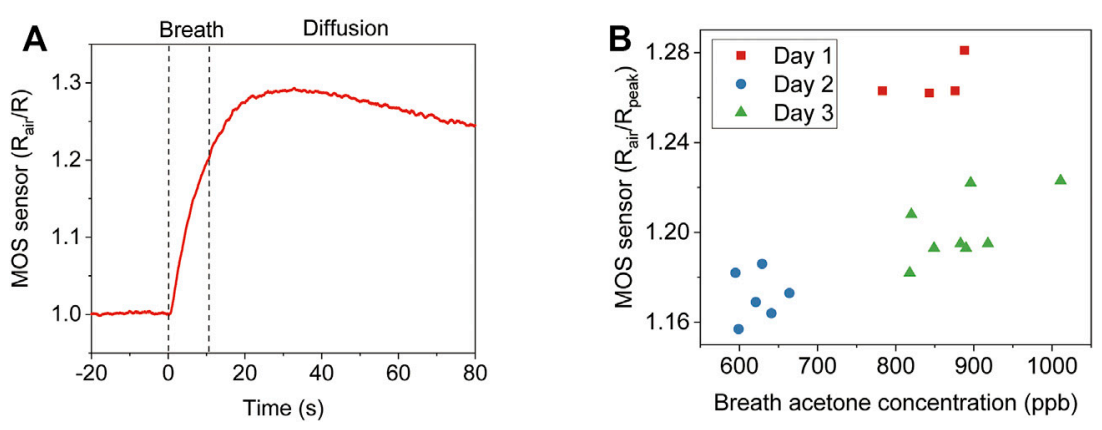

FIGURE 2|Direct analysis of breath acetone using the peak response of a MOS sensor. (A) The MOS sensor signal of one online breath test. (B) Comparison of the MOS sensor peak response and SIFT-MS analysis to the breath samples of one subject for 3 days.

in multiple ion monitoring (MIM) modes. $\mathrm{H} 3 \mathrm{O}+(\mathrm{m} / \mathrm{z}$ : 19) was chosen as the precursor ion for reaction with breath samples. The diffusive gas separation device was directly connected to the SIFT-MS injector (Supplementary Figure S1), so that a breath sample can be tested by both the acetone sensor and the SIFT-MS.

\section{RESULT AND DISCUSSION}

\section{Principle of the Device}

To collect a breath sample, a human subject directly breathes into the T-piece for about $10 \mathrm{~s}$ to trap the end portion of the breath. During exhalation, the elastic membranes of the check valves are bent by the breath pressure, allowing the breath to pass through (Figure 1A). After exhalation stops, the two elastic membranes restore their original flat shape (Figure 1B), which forms a breath reservoir, traps the breath sample, and prevents reverse airflow caused by inhalation. Captured gas molecules can escape from the reservoir through the small gaps between the elastic membrane and its holder (Figure 1C), and different types of gas molecules escape at different speeds because of different diffusion coefficients. Thus, the ratio of acetone concentration to interference gases changes over time. By optimizing the diffusion and measurement time, acetone can be detected with improved accuracy.

\section{BrAce Measurement and Interference Analysis}

Figure 2A shows the signal of one online breath test. The MOS sensor responds immediately after exhalation starts. After the exhalation stops, it takes some additional time for the gas molecules to diffuse to the MOS sensor before peak response is reached. Because the gas molecules keep diffusing into the ambient air, the signal decreases after the peak response. We further conducted three consecutive real breath tests in Supplementary Figure S2, which showed a good consistency. An intuitive way of signal analysis is simply using the peak value. To evaluate the performance, we tested the breath samples of one subject for 3 days. A selected ion flow tube mass spectrometer (SIFT-MS) was used as a reference instrument. As shown in
Figure 2B, there is a clear offset between day 1 and day 3 . Since no alcohol was consumed, and the ambient humidity was similar for the 3 days, other interference gases in exhaled breath can be the cause of the offset.

To verify that the MOS sensor responds to other gas components in exhaled breath, a gas washing bottle filled with water was connected to the inlet to filter out acetone in breath. The removal of acetone by the bottle filter was verified by SIFTMS (Supplementary Figure S3). Figure 3A shows the results of two consecutive breath tests, one with a filter and one without. It can be observed that the MOS sensor showed a significant response to the filtered breath. The peak response shows up earlier and the signal decreases faster than the unfiltered breath, which indicates that the interference gases in the filtered breath have large diffusion coefficients. Hydrogen is probably the major interference component, which is not absorbed by water and has a large diffusion coefficient compared to acetone. Supplementary Figure S4 shows the cross-sensitivity of the used MOS acetone sensor. Although it is less sensitive to hydrogen than acetone, the hydrogen concentration in breath can increase up to $50 \mathrm{ppm}$. Hydrogen interference is a major issue not only because of its high concentration in exhaled breath but also because its presence is hard to control by diet like breath alcohol. We solve the hydrogen interference issue by using diffusive gas separation (Figure 3B). $790 \mathrm{ppb}$ acetone and $50 \mathrm{ppm}$ hydrogen were prepared representing a normal BrAce level and a high breath hydrogen level. The two samples were pumped through the T-piece separately. Although the MOS sensor's response to $50 \mathrm{ppm}$ hydrogen was much higher than $790 \mathrm{ppb}$ acetone, the response of the MOS sensor to hydrogen decreased much faster than acetone after sampling stopped. At $130 \mathrm{~s}$, almost no hydrogen can be detected, but the response of the MOS sensor to acetone is still about $60 \%$ of the peak response. In addition, we further confirmed the diffusion coefficients of acetone-air $\left(0.124 \mathrm{~cm}^{2} \mathrm{~s}^{-1}\right)$ and hydrogen-air $\left(0.627 \mathrm{~cm}^{2} \mathrm{~s}^{-1}\right)$ under the circumstance $(p=101.325 \mathrm{kPa}, \mathrm{T}=293.15 \mathrm{~K}$ ) (Marrero and Mason, 1972).

Conventionally, MOS acetone sensors are calibrated and tested using synthetic gas samples, but this doesn't translate into accurate acetone detection in real breath samples, considering the complex components in exhaled breath. To 

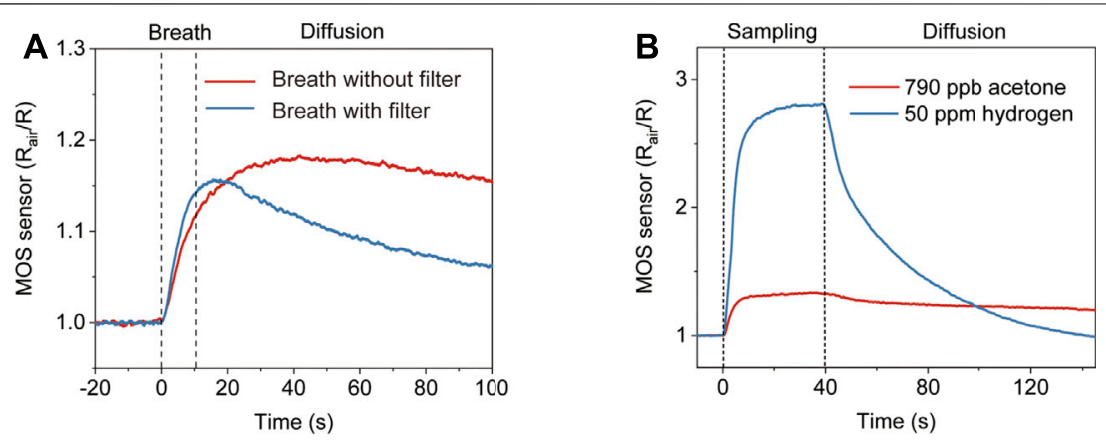

FIGURE 3 | The response of the diffusive gas separation device to compounds with different diffusion coefficients. (A) The response of the device to two consecutive breath tests with/without the water filter. (B) The response of the device to 790 ppb acetone with larger diffusion coefficients and 50 ppm hydrogen with smaller diffusion coefficients, respectively.
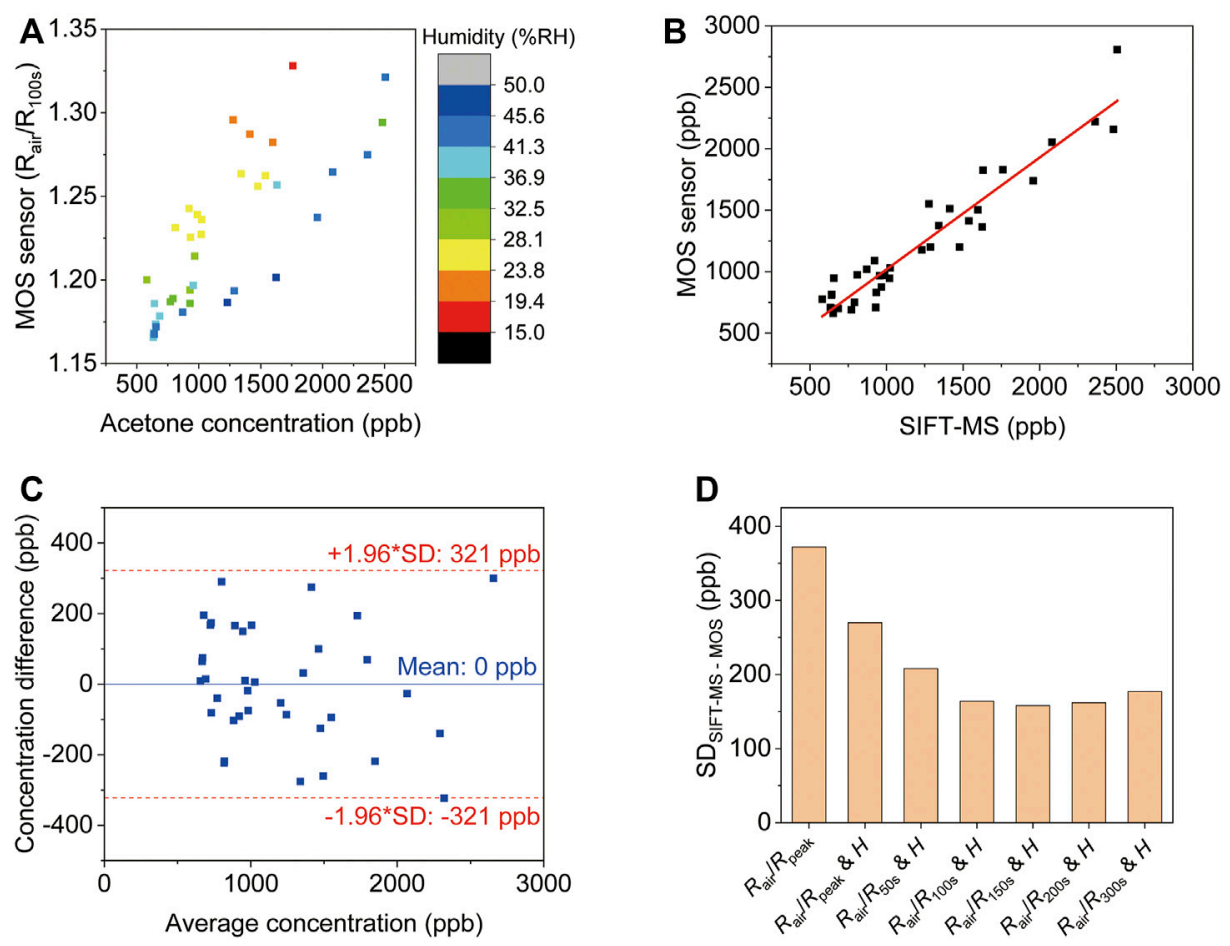

FIGURE 4 | Analysis of breath acetone using the diffusive gas separation device. (A) The MOS sensor signals of all sample tests at $100 \mathrm{~s}$ ( 0 s indicates the start of breath sampling). The different color of the data points denotes the initial humidity level in the reservoir. The acetone concentrations of the samples were evaluated via SIFT-MS. (B) The linear correlation between MOS sensor response corrected using Eq. 1 and the breath acetone concentrations $(R=0.95, p<0.001)$. (C) The limit of agreement between the device and the SIFT-MS. (D) The standard deviation of the difference between SIFT-MS and MOS using different correction parameters.

calibrate the device, 37 real breath samples were tested in the same way as real application scenarios, i.e., directly breath into the device for $10 \mathrm{~s}$. Humidity interference is another common issue for gas sensing. Although the humidity level in the reservoir is always $100 \% \mathrm{RH}$ after breath sampling, it can be different before each test, so a humidity sensor was placed in the reservoir to record the initial humidity level. The acetone concentration of each breath sample was confirmed by SIFT-MS. Figure 4A shows the MOS sensor signals of all the tests at $100 \mathrm{~s}(0 \mathrm{~s}$ indicates the start of breath sampling). The color of each data point represents the initial humidity level in the reservoir, which clearly has an impact on the response of the MOS sensor. A multiple linear regression equation was used to predict acetone concentration:

$$
[\text { Acetone }]=A_{1}+A_{2} \times\left(\frac{R_{\text {air }}}{R_{100 s}}\right)+A_{3} \times H
$$

Where [Acetone] is the acetone concentration, $R_{\text {air }}$ is the resistance of the MOS sensor before breath sampling, $R_{100 \mathrm{~s}}$ is the resistance of the MOS sensor $100 \mathrm{~s}$ after breath sampling 


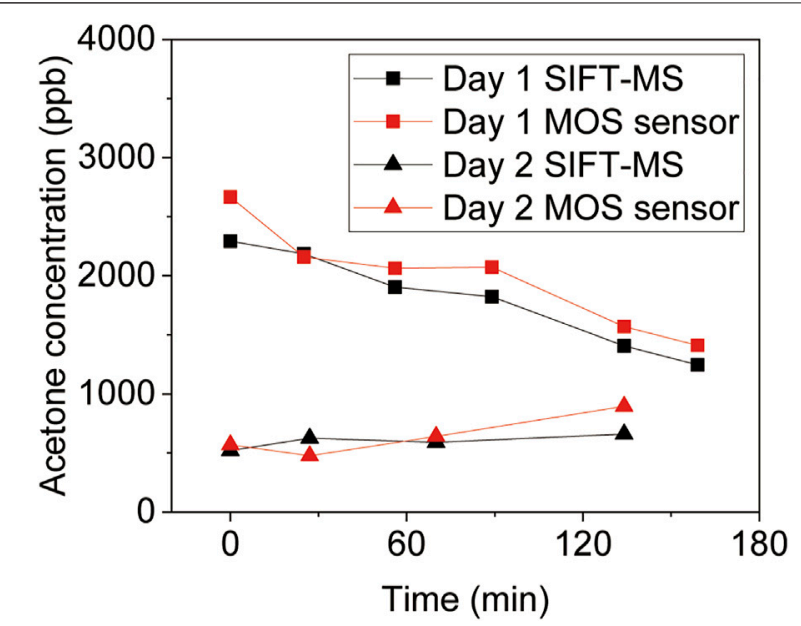

FIGURE 5 | BrAce based diet tracking using diffusive gas separation device. The volunteer received a high fat and low carbohydrate dinner the day before Day 1, and a high carbohydrate breakfast about $4 \mathrm{~h}$ before the test. In addition, the volunteer received a normal diet on Day 2 as a comparison.

starts, and $H$ is the initial humidity level. Figure $4 \mathbf{B}$ shows that the BrAce concentrations obtained from our device and the SIFT-MS have a strong correlation $(R=0.95, p<0.001)$. The best fitting values of A1, A2 and A3 for Eq. 1 are given in Supplementary Table S1.

The limit of agreement between the device and the SIFT-MS was determined from a Bland-Altman plot as shown in Figure 4C. The difference between the readings from the MOS sensor and the corresponding reading from SIFT-MS was plotted against the average reading of SIFT-MS and the MOS sensor. The limit of agreement was \pm 1.96 standard deviations (SD) from the mean, which was $321 \mathrm{ppb}$. This means that within $95 \%$ confidence the reading from the MOS sensor is within $321 \mathrm{ppb}$ difference from the reading obtained from SIFT-MS. Regression equations using MOS signals at different time points were compared to show the benefits of the used approach (Figure 4D). Simply using the peak response value leads to the largest variation between the MOS sensor and SIFT-MS. Including humidity signal can reduce the variation. The smallest variation is obtained using $R_{150 \mathrm{~s}}(\mathrm{SD}=158 \mathrm{ppb})$. This allows accurate detection of low concentration BrAce.

\section{BrAce Based Diet Tracking}

As a demo application, here we show that the device can be used to track diet (Figure 5). On day 1, the initial high BrAce level of a healthy male subject was reached via a high fat and low carbohydrate dinner the day before. A high carbohydrate breakfast about $4 \mathrm{~h}$ before the test was consumed to switch body energy source from fat to carbohydrate. It is noteworthy that eating high carb content will make bacterias in the gut produce methane from breaking down the carbs. However, the sensor (SB-33) employed in this study is much more sensitive to acetone than methane as shown in Supplementary Figure S4 in Supplementary material. As expected, a continuous decrease of BrAce was detected by both the device and SIFT-MS. A control
TABLE 1 | Comparison with recent studies of MOS acetone sensors.

\begin{tabular}{|c|c|c|c|}
\hline Sensor & Sample & Sample delivery & Ref \\
\hline $\mathrm{Pt}-\mathrm{WO}_{3}$ & real breath & direct exhalation & This work \\
\hline $\mathrm{PdO}-\mathrm{Co}_{3} \mathrm{O}_{4}$ & spiked breath & air bag/pump & Koo et al. (2017) \\
\hline $\mathrm{Si}-\mathrm{WO}_{3}$ & real breath & pump & Güntner et al. (2017) \\
\hline $\mathrm{Co}_{3} \mathrm{O}_{4}$ & synthetic gas & liquid evaporation & Zhang et al. (2015) \\
\hline Ir-GO- $\mathrm{Co}_{3} \mathrm{O}_{4}$ & synthetic gas & gas injection & Choi et al. (2014) \\
\hline $\mathrm{GO}-\mathrm{ZnO}$ & synthetic gas & liquid evaporation & Wang et al. (2016) \\
\hline $\mathrm{Pt}-\mathrm{WO}_{3}$ & synthetic gas & mass flow controller & Shin et al. (2013) \\
\hline Pt-PS-SnO 2 & spiked breath & air bag/pump & Jang et al. (2016) \\
\hline $\mathrm{GO}-\mathrm{SnO}_{2}$ & real breath & air bag/syringe & Kalidoss et al. (2019) \\
\hline $\mathrm{ZnO}-\mathrm{Co}_{3} \mathrm{O}_{4}$ & synthetic gas & syringe injection & Zhang et al. (2019) \\
\hline Pt-SnO 2 & synthetic gas & mass flow controller & Cho et al. (2020) \\
\hline
\end{tabular}

experiment performed on day 2 shows the detection of basal BrAce under normal diet conditions. The good correlation and agreement between the device and SIFT-MS validate its reliability in the detection of real breath samples. Table 1 compares this work with recent studies of MOS acetone sensors, showing its unique feature of online breath sampling, yet with the capability of quantifying low-level BrAce.

\section{CONCLUSION}

In conclusion, we proposed a simple device and novel strategy for online and accurate detection of BrAce. With a breath reservoir and two check valves, the exhaled gas can be sampled and separated in $100 \mathrm{~s}$, and the influences of other interference gases in breath are significantly reduced. Moreover, we further optimized the diffusion time, corrected humidity interference, so that the device showed an excellent consistency with the SIFT-MS in real breath samples measurement. Finally, we employed the device to track BrAce of human subjects, and the results were consistent with standard instruments and can indicate different diet conditions. Compared to standard instrument-based methods, our devices showed significant advantages in complexity and cost, which enabled a simple and accurate online measurement of BrAce with conventional MOS sensors. Considering the significance of BrAce monitoring for human health, we anticipate our proposed device would promote the realization of personal monitoring of fat metabolism, provide insights into health status, and prevent various chronic diseases.

\section{DATA AVAILABILITY STATEMENT}

The original contributions presented in the study are included in the article/Supplementary Material, further inquiries can be directed to the corresponding authors.

\section{AUTHOR CONTRIBUTIONS}

DW, HD, and LQ designed the study. HD, LQ, YC, and XZ performed experiments and analyzed data. CC, QC, FX, and JW 
provided an experimental resource. DW, XC, HD, and LQ wrote and revised the manuscript.

\section{FUNDING}

The authors sincerely acknowledge financial support from the Major Scientific Project of Zhejiang Lab (No. 2020MC0AD01), Open Project of Zhejiang Lab (No. 2021MC0AB02), National Natural Science Foundation of China (Grant No. 82172064),

\section{REFERENCES}

Alizadeh, N., Jamalabadi, H., and Tavoli, F. (2019). Breath Acetone Sensors as Non-invasive Health Monitoring Systems: A Review. IEEE Sensors J. 20 (1), 5-31. doi:10.1109/JSEN.2019.2942693

Anderson, J. C. (2015). Measuring Breath Acetone for Monitoring Fat Loss: Review. Obesity 23 (12), 2327-2334. doi:10.1002/oby.21242

Chen, L., Song, Y., Liu, W., Dong, H., Wang, D., Liu, J., et al. (2022). MOFbased Nanoscale Pt Catalyst Decorated SnO2 Porous Nanofibers for Acetone Gas Detection. J. Alloys Compd. 893, 162322. doi:10.1016/j. jallcom.2021.162322

Chen, X., Muhammad, K. G., Madeeha, C., Fu, W., Xu, L., Hu, Y., et al. (2021). Calculated Indices of Volatile Organic Compounds (VOCs) in Exhalation for Lung Cancer Screening and Early Detection. Lung Cancer 154, 197-205. doi:10.1016/j.lungcan.2021.02.006

Chen, X., Wang, F., Lin, L., Dong, H., Huang, F., Ghulam Muhammad, K., et al. (2017). Association of Smoking with Metabolic Volatile Organic Compounds in Exhaled Breath. Ijms 18 (11), 2235. doi:10.3390/ ijms 18112235

Cho, H. J., Choi, S.-J., Kim, N.-H., and Kim, I.-D. (2020). Porosity Controlled 3D SnO2 Spheres via Electrostatic spray: Selective Acetone Sensors. Sensors and Actuators B-Chemical 304, 127350. doi:10.1016/j.snb.2019. 127350

Choi, S.-J., Ryu, W.-H., Kim, S.-J., Cho, H.-J., and Kim, I.-D. (2014). Bifunctional Co-sensitization of Graphene Oxide Sheets and Ir Nanoparticles on P-type Co3O4 Nanofibers for Selective Acetone Detection. J. Mater. Chem. B 2 (41), 7160-7167. doi:10.1039/c4tb00767k

Dong, H., Zhang, F., Chen, J., Yu, Q., Zhong, Y., Liu, J., et al. (2020). Evaluating Propofol Concentration in Blood from Exhaled Gas Using a BreathingRelated Partition Coefficient. Anesth. Analgesia 130 (4), 958-966. doi:10. 1213/ane.0000000000004225

Dong, H., Zhang, F. J., Wang, F. Y., Wang, Y. Y., Guo, J., Kanhar, G. M., et al. (2017). Simultaneous On-Line Monitoring of Propofol and Sevoflurane in Balanced Anesthesia by Direct Resistive Heating Gas Chromatography. J. Chromatogr. A 1506, 93-100. doi:10.1016/j. chroma.2017.05.001

Güntner, A. T., Sievi, N. A., Theodore, S. J., Gulich, T., Kohler, M., and Pratsinis, S. E. (2017). Noninvasive Body Fat Burn Monitoring from Exhaled Acetone with Si-Doped WO3-Sensing Nanoparticles. Anal. Chem. 89 (19), 10578-10584. doi:10.1021/acs.analchem.7b02843

Jang, J.-S., Choi, S.-J., Kim, S.-J., Hakim, M., and Kim, I.-D. (2016). Rational Design of Highly Porous SnO2Nanotubes Functionalized with Biomimetic Nanocatalysts for Direct Observation of Simulated Diabetes. Adv. Funct. Mater. 26 (26), 4740-4748. doi:10.1002/adfm.201600797

Kalidoss, R., Umapathy, S., Anandan, R., Ganesh, V., and Sivalingam, Y. (2019). Comparative Study on the Preparation and Gas Sensing Properties of Reduced Graphene Oxide/SnO2 Binary Nanocomposite for Detection of Acetone in Exhaled Breath. Anal. Chem. 91 (8), 5116-5124. doi:10.1021/ acs.analchem.8b05670

King, J., Kupferthaler, A., Unterkofler, K., Koc, H., Teschl, S., Teschl, G., et al. (2009). Isoprene and Acetone Concentration Profiles during Exercise on
Zhejiang Provincial Natural Science Foundation of China (No. LQ21F010003), China Postdoctoral Science Foundation (No. 2020M681952).

\section{SUPPLEMENTARY MATERIAL}

The Supplementary Material for this article can be found online at: https://www.frontiersin.org/articles/10.3389/fbioe.2022.861950/ full\#supplementary-material

an Ergometer. J. Breath Res. 3 (2), 027006. doi:10.1088/1752-7155/3/2/ 027006

Koo, W.-T., Yu, S., Choi, S.-J., Jang, J.-S., Cheong, J. Y., and Kim, I.-D. (2017). Nanoscale PdO Catalyst Functionalized Co3O4 Hollow Nanocages Using MOF Templates for Selective Detection of Acetone Molecules in Exhaled Breath. ACS Appl. Mater. Inter. 9 (9), 8201-8210. doi:10.1021/acsami. $7 \mathrm{~b} 01284$

Liang, Y., Wu, Z., Wei, Y., Ding, Q., Zilberman, M., Tao, K., et al. (2022). Self-Healing, Self-Adhesive and Stable Organohydrogel-Based Stretchable Oxygen Sensor with High Performance at Room Temperature. Nano-micro Lett. 14 (1), 52. doi:10.1007/s40820-02100787-0

Marrero, T. R., and Mason, E. A. (1972). Gaseous Diffusion Coefficients. J. Phys. Chem. Reference Data 1 (1), 3-118. doi:10.1063/1.3253094

Musa-Veloso, K., Likhodii, S. S., and Cunnane, S. C. (2002). Breath Acetone Is a Reliable Indicator of Ketosis in Adults Consuming Ketogenic Meals. Am. J. Clin. Nutr. 76 (1), 65-70. doi:10.1093/ajcn/76.1.65

Pauling, L., Robinson, A. B., Teranishi, R., and Cary, P. (1971). Quantitative Analysis of Urine Vapor and Breath by Gas-Liquid Partition Chromatography. Proc. Natl. Acad. Sci. 68 (10), 2374-2376. doi:10.1073/ pnas.68.10.2374

Phillips, M., Gleeson, K., Hughes, J. M. B., Greenberg, J., Cataneo, R. N., Baker, L., et al. (1999). Volatile Organic Compounds in Breath as Markers of Lung Cancer: a Cross-Sectional Study. The Lancet 353 (9168), 1930-1933. doi:10. 1016/s0140-6736(98)07552-7

Schwarz, K., Pizzini, A., Arendacká, B., Zerlauth, K., Filipiak, W., Schmid, A., et al. (2009). Breath Acetone-Aspects of normal Physiology Related to Age and Gender as Determined in a PTR-MS Study. J. Breath Res. 3 (2), 027003. doi:10.1088/1752-7155/3/2/027003

Shin, J., Choi, S.-J., Lee, I., Youn, D.-Y., Park, C. O., Lee, J.-H., et al. (2013). Thin-Wall Assembled SnO2Fibers Functionalized by Catalytic Pt Nanoparticles and Their Superior Exhaled-Breath-Sensing Properties for the Diagnosis of Diabetes. Adv. Funct. Mater. 23 (19), 2357-2367. doi:10. 1002/adfm.201202729

Španěl, P., Dryahina, K., Rejšková, A., Chippendale, T. W. E., and Smith, D. (2011). Breath Acetone Concentration; Biological Variability and the Influence of Diet. Physiol. Meas. 32 (8), N23-N31. doi:10.1088/09673334/32/8/N01

Stefano, K., Rosalia, A., Chiara, B., Federica, G., Marco, M., Gioacchino, L., et al. (2018). Non-invasive Tests for the Diagnosis of helicobacter Pylori: State of the Art. Acta Bio Med. Atenei Parmensis 89 (Suppl. 8), 58. doi:10. 23750/abm.v89i8-S.7910

Wang, D., Zhang, F., Prabhakar, A., Qin, X., Forzani, E. S., and Tao, N. (2020). Colorimetric Sensor for Online Accurate Detection of Breath Acetone. ACS Sens. 6 (2), 450-453. doi:10.1021/acssensors.0c02025

Wang, P., Wang, D., Zhang, M., Zhu, Y., Xu, Y., Ma, X., et al. (2016). ZnO Nanosheets/graphene Oxide Nanocomposites for Highly Effective Acetone Vapor Detection. Sensors Actuators B: Chem. 230, 477-484. doi:10.1016/j. snb.2016.02.056

Wang, Z., and Wang, C. (2013). Is Breath Acetone a Biomarker of Diabetes? A Historical Review on Breath Acetone Measurements. J. Breath Res. 7 (3), 037109. doi:10.1088/1752-7155/7/3/037109 
Wu, Z., Rong, L., Yang, J., Wei, Y., Tao, K., Zhou, Y., et al. (2021). IonConductive Hydrogel-Based Stretchable, Self-Healing, and Transparent NO2 Sensor with High Sensitivity and Selectivity at Room Temperature. Small 17 (52), e2104997. doi:10.1002/smll.202104997

Zhang, D., Yang, Z., Wu, Z., and Dong, G. (2019). Metal-organic Frameworks-Derived Hollow Zinc Oxide/cobalt Oxide Nanoheterostructure for Highly Sensitive Acetone Sensing. Sensors Actuators B: Chem. 283, 42-51. doi:10.1016/j.snb.2018.11.133

Zhang, Z., Wen, Z., Ye, Z., and Zhu, L. (2015). Gas Sensors Based on Ultrathin Porous $\mathrm{Co} 3 \mathrm{O} 4$ Nanosheets to Detect Acetone at Low Temperature. RSC Adv. 5 (74), 59976-59982. doi:10.1039/c5ra08536e

Conflict of Interest: The authors declare that the research was conducted in the absence of any commercial or financial relationships that could be construed as a potential conflict of interest.
Publisher's Note: All claims expressed in this article are solely those of the authors and do not necessarily represent those of their affiliated organizations, or those of the publisher, the editors and the reviewers. Any product that may be evaluated in this article, or claim that may be made by its manufacturer, is not guaranteed or endorsed by the publisher.

Copyright $\odot 2022$ Dong, Qian, Cui, Zheng, Cheng, Cao, Xu, Wang, Chen and Wang. This is an open-access article distributed under the terms of the Creative Commons Attribution License (CC BY). The use, distribution or reproduction in other forums is permitted, provided the original author $(s)$ and the copyright owner(s) are credited and that the original publication in this journal is cited, in accordance with accepted academic practice. No use, distribution or reproduction is permitted which does not comply with these terms. 